\title{
Identification of cases of pododermatitis in broiler chickens when feeding a probiotic feed additive
}

\author{
Ivan Koshchaev ${ }^{1,{ }^{*}}$, Kristina Mezinova ${ }^{1}$, Antonina Ryadinskaya $^{1}$, Nadezhda Sorokina ${ }^{1}$, and \\ Sergei Chuev ${ }^{1}$ \\ ${ }^{1}$ Belgorod State Agricultural University, 308503, Vavilova str., 1., Maisky, Belgorod region, \\ Belgorod oblast, Russia
}

\begin{abstract}
Pododermatitis is a major concern in broiler production and is used as an audit criterion for poultry health checks in the United States and Europe. Pododermatitis is often associated with wet litter or poor litter quality. It is considered painful for birds and reduces the number of times the affected bird approaches the feeders and drinkers. Reduced feed and water consumption has a significant impact on weight gain, feed conversion and overall herd performance. As a result of the studies carried out to assess the degree of pododermatitis, it was observed that the quality of the paws increases or decreases, depending on the composition of the feed consumed.
\end{abstract}

\section{Introduction}

Poultry farming as one of the knowledge-intensive and dynamically developing branches of animal husbandry occupies a leading place in the supply of food to the population, providing one third of the protein part of animal origin in the human diet. [1]

In a difficult period of development of poultry farming in our country, scientists and poultry farmers are faced with the task of increasing the safety and productivity of poultry, improving the quality of products at the lowest material costs. In solving this problem, an important place belongs to biological methods of increasing productivity - improving feeding using biologically active substances of various origins. [2]

In an industrial setting, broiler chickens are exposed to constant stress factors that can increase their susceptibility to intestinal diseases, leading to reduced productivity, suppression of immunity and increased mortality. One of the typical causes of imbalance in the intestinal microflora is an increase in the content of undigested nutrients in the intestinal lumen. Clostridium Perfringens - a bacterium that is usually present in the intestines of poultry in an amount below $105 \mathrm{CFU}$ per gram of intestinal contents in a normal state without negative impact on the health of the bird. Virulent properties of Clostridium Perfringens begin to show when its population increases to 107-109 CFU per gram of intestinal contents. A high level of feed protein, in turn, is recognized as a factor contributing to the development of Clostridium Perfringens, and especially its strains capable of producing NetB-toxin, which is considered the main factor in the development of necrotic enteritis in broilers. [3]

Antibiotics are widely used to maintain and improve productivity in such conditions. However, in recent years, there has been a tightening of the rules on the use of antibiotics in animal husbandry in order to stop them from entering food for humans, as well as in

\footnotetext{
*Corresponding author:koshchaev@yandex.ru
} 
connection with the emergence of strains of pathogenic bacteria that have developed resistance to a wide range of antibiotics, thereby posing a health hazard and human life. In this regard, manufacturers are looking for alternatives to antibiotics. The range of potential substitutes is very wide. One of the most promising groups is probiotics. [4]

The role of probiotic crops in increasing the productivity of agricultural birds are common knowledge. The biological role and practical use of probiotics in the nutrition of agricultural many studies are devoted to birds.

The scientific literature contains data on the successful use of probiotics to increase the resistance of the organism of animals and birds.

The spectrum of indications for the use of probiotics is wide: they are used to stimulate cellular and humoral immunity factors, activate metabolic processes and normalize digestion, treat and prevent dysbiosis, gastrointestinal diseases of infectious and alimentary etiology, normalize the microflora of the digestive tract after treatment with antibiotics and other antibacterial chemotherapy. means to stimulate the growth of young animals, accelerate the adaptation of animals to high-energy diets and non-protein nitrogenous substances, increase the efficiency of feed use. [5]

It has been established that the range of probiotic use in clinical practice can be significantly expanded. They are used to stimulate nonspecific immunity, prevent and treat mixed gastrointestinal infections, digestive disorders of alimentary etiology (dysbacteriosis, acute lactic acidosis, etc.) arising from a sharp change in the composition of the diet, feeding disorders, technological stress and other reasons; to re-establish the microflora of the digestive tract after treatment with antibiotics and other antibacterial chemotherapeutic agents; replacing antibiotics in compound feed for young animals, improving digestion processes; accelerating adaptation to high-energy diets and non-protein nitrogenous substances; increasing the efficiency of feed use and poultry productivity. [6]

Pododermatitis is a major concern in broiler production and is used as an audit criterion for poultry health checks in the US and Europe. But many manufacturers pay more attention to other indicators. They feel that it doesn't make sense to spend extra money on paw health when that resource can be spent on increasing weight gain or improving feed conversion rates. The main thing is to sell meat, not legs. This opinion prevails in enterprises, regardless of whether $90 \%$ or $10 \%$ of the livestock suffer from paw disease.

Outdoor maintenance activates the vital activity of parasites that cause various diseases in chickens. Therefore, the company has additional costs for medicines, which are later found in meat and eggs. The activity of poultry with cage content is limited, therefore, the average daily gain and slaughter yield of poultry meat increases. [7]

Broilers go to the feeders and drinkers many times every day. They constantly stain the litter, which reacts to the condition of the paws. Pododermatitis is often associated with wet litter or poor litter quality. It is considered painful for birds and reduces the number of times the affected bird approaches the feeders and drinkers. When pain occurs, the bird restricts its movement until the wound heals. Reduced feed and water consumption have a significant impact on weight gain, feed conversion and overall flock performance.

Today producers have come to the conclusion that increased litter moisture not only causes pododermatitis, but also reduces broiler productivity, marketable carcass weight and has a negative impact on other aspects of poultry management. Anything that is detrimental to productivity reduces the value of the sales proceeds. Therefore, even if it seems that producers are not paying for "healthy feet", they are actually getting costs cleverly disguised as the cost of gaining weight gain and improving feed conversion.

Contact dermatitis affects areas of the skin that have prolonged contact with the bedding: the paw pads, the back of the tendons and, in severe cases, the breast area. It is noted by the development in broilers and turkeys, as well as in parent flocks. Contact dermatitis is usually detected when assessing the general condition of the body: from 
individual surface lesions to deep ulcers of various sizes at different stages and severity of the lesion, which affect the degree of discomfort and pain shock. [8]

Symptoms:

- Blackening of skin, progressing to erosion, fibrosis of footpad surface, damage to the tendons, and sometimes namina on the breast.

- Causes lameness.

- Serves as an "entrance gate" for secondary infections.

Causes:

- Poor quality litter material, including high contamination with bacteria, mold, etc.

- The state of the intestine, the presence of dysbiosis, which affects:

- High humidity of droppings and further on the quality of litter.

- Impaired intake of minerals and vitamins necessary for the formation of bones and joints in the bird's body.

- Incorrect operation of drinking system.

- Incorrect operation of ventilation system based on maintaining the level of relative humidity and ammonia

Since pododermatitis is closely related to the quality of the litter, the manifestation of the disease also affects other aspects of the general health of the bird, for example, gait, the appearance of nests on the breast and tendon burns. Litter quality has never been a major concern as it is today. This gives manufacturers a reason to rethink how much attention should be paid to this factor. It was believed that updating the litter is the ideal way out of the situation. However, this is not the case, and the consequences appear in some other situations. In fact, today, in most cases, spring cleaning and new litter will still result in poor paw quality in the first two or three litter plantings from the very first flock, often causing damage to the paws of everyone else.

Much attention is mainly focused on improving the condition of the paws at the end of the fattening period, as the slaughter age approaches, but the damage to the paws begins much earlier, even in the first week of life. The delicate legs of chicks are damaged by sharp edges, large pieces and fragments of poor-quality litter material. [9]

In addition, the high cost of litter means that producers can save money by returning the updated litter back to the house. However, adding less than $10 \mathrm{~cm}$ of fresh litter will likely not be sufficient for renewal. Add to that the fact that growers ventilate the bedding, although ammonia is generally not an issue. Unfortunately, airing the litter is not a cure-all for dampness and increased moisture levels in the house that quickly moistens the litter and further damages paws.

In addition, poultry excrete uric acid in their droppings, which is converted to dissolved ammonia through a fermentation reaction. This ammonia is corrosive and can "burn" the footpad. Burns arise from the combination of high levels of humidity and high levels of ammonia, as well as damage from other chemical compounds that are contained in the droppings, upon contact with it.

The problem will be exacerbated in the current broiler production environment if the appropriate measures are not taken. The high stocking density and high productivity of new crosses increases the risk of injury. Today, poultry is raised at high weights to meet the growing demand for high quality at an affordable price, with further processing and production of instant chicken products. This means birds stay in the brood for much longer, resulting in high litter moisture in the absence of proper ventilation control.

While the new litter materials present their share of paw health problems for the first two flocks, older materials also have drawbacks if not handled properly. Breeders may be able to remove a large amount of old litter between new flocks leaving less than $10 \mathrm{~cm}$ on the floor, not enough to properly cushion / isolate and absorb moisture. In this case, the old litter becomes damp and crumples, and the same problems arise as when using a lower 
quality material of the new litter layer. The problem is exacerbated by the fact that more ammonia remains on the floor in the old litter than in the new one. [10]

But ammonia isn't the only cause of poor paw health. Wet litter by itself can cause sores on the feet. Moisture softens the paw pads, making them more susceptible to injury and leading to dermatitis. This is often seen when using new litter materials with inadequate ventilation, when the relative humidity remains high (with low ammonia levels) and makes the entire litter moist. [11]

Another problem occurs when there is a lot of litter on the floor. If the litter is too deep (more than 15-20 cm), it becomes difficult to penetrate into a formation near the floor covering. This hard layer will become a source of moisture and generate huge amounts of ammonia into the house and heated bedding prepared for the chicks. The increased release of ammonia will not only threaten the bird's paws but also the eyes and respiratory system. The high concentration of ammonia is also a threat to the eyes and respiratory system of plant workers.

Many producers now rake the bedding between flock changes to dry and break up the hard bed. It is important to properly rake the entire hard layer down to the floor and remove as much dirt as possible in order to dry everything properly. However, you need to be careful not to remove more than expected.

This preparation takes time and equipment, but has advantages over simple litter change, as long as all regulations and temperature conditions are met. $54^{\circ} \mathrm{C}$. Many integrating companies are encouraging their producers to do this before planting and to work with sufficient time to clean up. [12]

It is important to consider that the concentration and cleanliness of litter can vary greatly at one end of the house from the other. The dividing grid divides the stands into four quadrants, and each quadrant can have different bedding qualities. Especially in warm weather, when cooler cages are used more often, where the litter will be more moist and caked than in other sectors. The ventilated quadrant will be drier if no humidifiers are used. In this case, litter in ventilated cages can become wet when some of the vapor is drawn to the rear of the house and escaped through the ventilation. [13]

This difference in bedding quality results in different paw conditions throughout the house. In most cases, the drier the litter, the better the paw quality. However, during the hot season, when birds are densely planted, it is difficult to maintain adequate litter moisture. During such periods, it is necessary to maintain a comfortable state of the bird, given the difficulties in maintaining the quality of the litter. This means that bedding between flock changes becomes even more important. Drying between flock changes will be critical to a successful start when planting a new flock. In excessive moisture conditions when new flocks start to plant, it will be difficult to maintain litter quality due to flock age. [14]

It is likely that more attention will be paid to litter quality control in the near future. In the not so distant past, litter did not rank high on the list of problems for producers. However, due to the emerging demand for poultry feet in the poultry market, which practically did not exist 20 years ago, and the increased attention to animal welfare issues, litter quality is now considered a priority issue. Birds have been in contact with litter on the floor for almost their entire life.

The type, quantity and quality of litter have a huge impact on bird performance. It is the performance indicators that determine how serious the bird has passed before the implementation stage. Thus, broiler producers to some extent pay for the quality of the condition of the poultry paws, sometimes without realizing this fact. [15]

\section{Materials and research methods}

Scientific and economic experience on the influence of probiotic cultures on the 
development of pododermatitis in broiler chickens was carried out on chickens of the Cobb500 cross in the conditions of the educational and scientific poultry farm of the UNITS "Agrotechnopark" of the Belgorod State Agrarian University.

From a batch of chickens of one hatch at one day old, 36 groups of 65 heads each were formed. A total of 4 different diets were studied, i.e. 9 groups (replicates) were fed for each diet. The experiment lasted 40 days.

Microclimate parameters, stocking density, feeding and drinking area were similar for all groups of poultry and corresponded to the normative indicators.

The poultry received diets of the Starter, Rost, Finisher brands. The bird received Starter food from the moment of setting on the experiment, on the 0th (1st) day. The transition from the Starter to the Growth brand feed was made at the age of 10 days by smoothly replacing one feed with another (in $\%$ of the standard - 70/30, 50/50, 30/70). Then the bird received food Growth up to 22 days of age. From 23 days of age, the bird was smoothly transferred to the Finisher feed (in a similar proportion as in the transition from Starter to Growth) and fed with this type of feed until the end of the experiment. The experiment scheme is presented in Table 1.

Features of feeding broiler chickens were as follows:

1 - Control group (Standard diet);

2 - Experienced group (Low protein diet);

3 - Experienced group (Standard diet + probiotic Bacillus amyloliquefaciens);

4 - Experienced group (Low protein diet + probiotic Bacillus amyloliquefaciens).

In experimental groups 2 and 4 (Low-protein diet), the addition of synthetic amino acids such as L-Valine, L-Isoleucine and L-arginine to the rations of GROWER and FINISHER. The reduction in the crude protein of the feed in groups 2 and 4 will be about $1.5-2 \%$ for each phase. The decrease in crude protein in these groups is limited by balancing the socalled. "Glycine equivalent" (the sum of glycine + serine x 0.7143 ), where the calculated value of this indicator is at least $118 \%$ of the total lysine.

Table 1. Scheme of the experiment with a uniform distribution of experimental groups in cells in the experimental building

\begin{tabular}{|c|c|c|c|}
\hline \multicolumn{5}{|c|}{ Diets / groups } \\
\hline T1 & T2 & T3 & T4 \\
\hline 11 & 21 & 31 & 41 \\
\hline 12 & 22 & 32 & 42 \\
\hline 13 & 23 & 33 & 43 \\
\hline 14 & 24 & 34 & 44 \\
\hline 15 & 25 & 35 & 45 \\
\hline 16 & 26 & 36 & 46 \\
\hline 17 & 27 & 37 & 47 \\
\hline 18 & 28 & 38 & 48 \\
\hline 19 & 29 & 39 & 49 \\
\hline
\end{tabular}

During the experiment, the following indicators were determined:

- microclimate data in the poultry house (daily) (temperature, humidity, ventilation parameters in $\mathrm{m} 3$ / $\mathrm{h}$ per head, illumination);

- feed distribution (daily);

- registration of diseases / waste / death (daily);

- cases of pododermatitis in poultry. 
The conditions of keeping chickens in all groups were the same and corresponded to the recommended standards for this cross.

\section{Research results and discussion}

Numerous studies have established that the conditions of keeping poultry often serve as stress factors for it, which reduce the efficiency of agricultural production.

The yield of quality poultry legs increases the profit of production. At slaughter age (40 days), cases of pododermatitis were counted on a scale from $0(\min )$ to $3(\max )$. The results are presented in tables 2,3,4.

The bird's legs were classified according to the methodology proposed by McWard and Taylor (2000) based on visual analysis and with the following scores: 0 - no damage; 1 minor lesion; 2 - moderate injury to one or both limbs; and 3 - extensive trauma to one or both limbs. (picture 1)

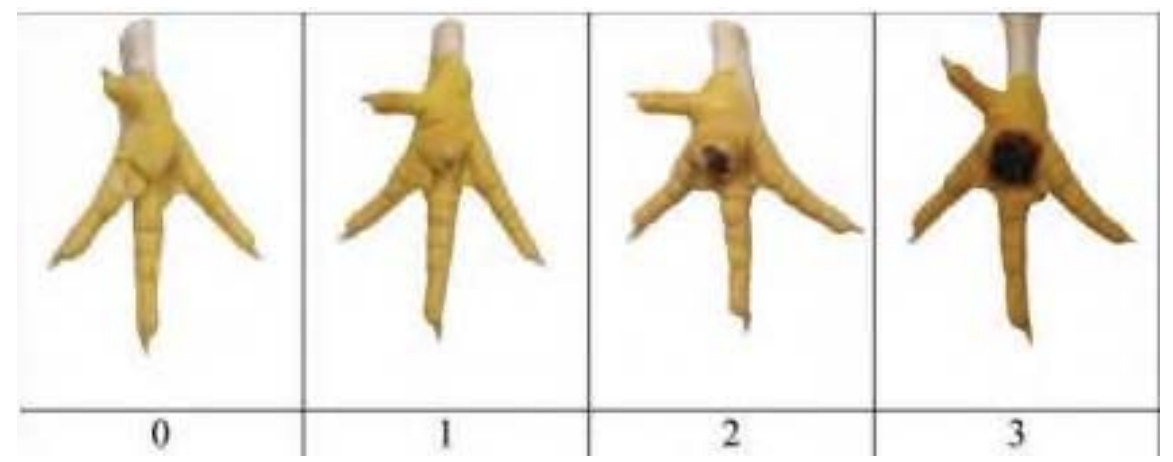

Fig. 1. Scoring scale for pododermatitis (ie, footpad disease) in broiler chickens.

Table 2. Cases of pododermatitis of chickens - broilers, at the age of 40 days, by groups 


\begin{tabular}{|c|c|c|c|c|c|}
\hline \multicolumn{2}{|l|}{ Group } & \multicolumn{4}{|c|}{ Cases of pododermatitis in poultry } \\
\hline & & $\mathbf{0}$ & 1 & 2 & 3 \\
\hline \multirow[t]{9}{*}{1} & 11 & 37 & 19 & 3 & \\
\hline & 12 & 39 & 22 & 1 & \\
\hline & 13 & 32 & 20 & 2 & 1 \\
\hline & 14 & 25 & 26 & 4 & \\
\hline & 15 & 33 & 18 & 1 & \\
\hline & 16 & 37 & 15 & 1 & \\
\hline & 17 & 28 & 20 & 6 & 1 \\
\hline & 18 & 37 & 16 & 1 & \\
\hline & 19 & 42 & 14 & & \\
\hline \multirow[t]{9}{*}{2} & 21 & 37 & 18 & 1 & \\
\hline & 22 & 42 & 12 & 1 & \\
\hline & 23 & 36 & 15 & 3 & \\
\hline & 24 & 44 & 11 & 2 & \\
\hline & 25 & 44 & 13 & & 1 \\
\hline & 26 & 36 & 17 & 1 & \\
\hline & 27 & 41 & 16 & 2 & \\
\hline & 28 & 41 & 14 & & \\
\hline & 29 & 38 & 13 & & \\
\hline \multirow[t]{9}{*}{3} & 31 & 35 & 21 & 1 & \\
\hline & 32 & 38 & 20 & & \\
\hline & 33 & 37 & 17 & 2 & \\
\hline & 34 & 39 & 16 & 3 & \\
\hline & 35 & 44 & 14 & 2 & \\
\hline & 36 & 44 & 15 & & 1 \\
\hline & 37 & 41 & 17 & 1 & \\
\hline & 38 & 36 & 22 & 1 & \\
\hline & 39 & 37 & 18 & 1 & \\
\hline \multirow[t]{9}{*}{4} & 41 & 44 & 12 & 1 & \\
\hline & 42 & 40 & 16 & & \\
\hline & 43 & 46 & 13 & 1 & \\
\hline & 44 & 48 & 10 & 2 & \\
\hline & 45 & 47 & 14 & & \\
\hline & 46 & 42 & 14 & 1 & \\
\hline & 47 & 50 & 9 & & \\
\hline & 48 & 38 & 19 & 1 & 1 \\
\hline & 49 & 47 & 10 & & \\
\hline
\end{tabular}

As a result of the studies carried out to assess the degree of pododermatitis, it was observed that the quality of the paws increases or decreases, depending on the composition of the feed consumed.

Among animals of the 1st (control) group, which received the standard diet, there was a higher incidence of injuries to the plantar pads, visually classified as 0 , the average result in the group 34, 4, compared with the 3rd group, which received the probiotic (39).

Group 4, who received a low-protein diet along with probiotics, showed a better result in assessing paw quality (44.6), compared with group 2 (39.9), who received a low-protein diet.

Comparing 1 (control) group and 2 group, it was noticed that a high frequency of manifestations of pododermatitis is noticeable in the control group. 
Thus, it can be concluded that the use of probiotics in low-protein diets of broilers reduces the degree of manifestation of pododermatitis.

Table 3. Cases of pododermatitis of chickens-broilers by ration, number

\begin{tabular}{|r|l|c|c|r|}
\hline \multirow{2}{*}{ Group } & \multicolumn{4}{|c|}{ Cases of pododermatitis in poultry } \\
\cline { 2 - 5 } & $\mathbf{0}$ & $\mathbf{1}$ & $\mathbf{2}$ & $\mathbf{3}$ \\
\hline $\mathrm{T} 1$ & 310 & 170 & 19 & 2 \\
\hline $\mathrm{T} 2$ & 359 & 129 & 10 & 1 \\
\hline $\mathrm{T} 3$ & 351 & 160 & 11 & 1 \\
\hline $\mathrm{T} 4$ & 402 & 117 & 6 & 1 \\
\hline
\end{tabular}

Table 4. Cases of pododermatitis in chickens-broilers by diet, \%

\begin{tabular}{|r|l|l|l|l|}
\hline \multirow{2}{*}{ Group } & \multicolumn{5}{|c|}{ Cases of pododermatitis in poultry, \% } \\
\cline { 2 - 5 } & $\mathbf{0}$ & $\mathbf{1}$ & $\mathbf{2}$ & $\mathbf{3}$ \\
\hline T1 & 61,9 & 33,9 & 3,8 & 0,4 \\
\hline T2 & 71,9 & 25,9 & 2,0 & 0,2 \\
\hline T3 & 67,1 & 30,6 & 2,1 & 0,2 \\
\hline T4 & 76,4 & 22,2 & 1,1 & 0,2 \\
\hline
\end{tabular}

In addition to the analysis of replicates of each of the groups during the study, a comparative analysis of the rations of feeding chickens - broilers was carried out (table 3, 4)

\section{Conclusions}

Comparing the standard (1) and standard ration + probiotic Bacillus amyloliquefaciens (3) rations for the purpose of detecting pododermatitis in broilers, we came to the conclusion that the pattern of manifestation of grade 0 pododermatitis in the given diets is as follows: standard ration - 310 heads $(61.9 \%)$, standard diet + probiotic Bacillus amyloliquefaciens 351 heads $(67.1 \%)$. It can be noted that the 0 degree of pododermatitis is higher in the diet with the use of the probiotic by 41 heads $(5.2 \%)$.

Comparison of a low-protein diet (2), where healthy paw pads were observed in 359 animals $(71.9 \%)$ with a low-protein diet + probiotic Bacillus amyloliquefaciens (4), where healthy paw pads were observed in 402 animals (76.4\%) showed that 0 the degree of pododermatitis is higher in the low-protein diet with the use of the probiotic by 43 heads $(4.5 \%)$, respectively.

The condition of the paw pads has become a major concern in the poultry industry in recent years. Manufacturers are less concerned with paw quality. This is because manufacturers think that there is no financial justification for improving foot condition. In fact, this is not the case.

If the poor condition of the poultry paws leads to a slowdown in growth rates, low weight gain, a decrease in productivity and a decrease in revenue, then this fact is a financial incentive for producers to improve the condition of the paws. This can be expressed in high carcass weights or low feed conversion, but certainly this aspect is present.

The quality of the litter largely determines the condition of the paws. Litter is not the only factor in paw health, but it is likely the most important.

Efforts to control optimal moisture, drinking system health, and litter $\mathrm{pH}$ are integral to maintaining good litter quality, improving poultry feet and optimizing flock performance. 


\section{References}

1. P.H. Selle, Animals, 4, 729 (2020)

2. V.Kh, Temiraev, Journal of Pharmaceutical Sciences and Research., 12, 2392-2396 (2017)

3. D. Sun, Pakistan Journal of Zoology, 5, 50 (2018)

4. O.A. Gracheva, International Journal of Research in Pharmaceutical Sciences, 1, 908$912(2020)$

5. V.Z. Khamitova, Zootechnics, 9,15-18 (2019)

6. A.A. Shaposhnikov, Research Result: Pharmacology and Clinical Pharmacology, 4,91-94 (2016)

7. V.I. Fisinin, Microbiology , 4, 493-499 (2016)

8. I.C. Jong, Appl. Poult. Res. 23, 51-58 (2014)

9. V.E. Michel, Brit. Poult. Sci. 53(3), 275-281 (2012)

10. J. Blair, Vet Clin Exot Anim. 16, 715-735 (2013)

11. E.E. Epimahova, Research Journal of Pharmaceutical, Biological and Chemical Sciences 6, 1774-1778 (2018)

12. S.I. Nikolayev, Advances in Animal and Veterinary Sciences 1,106-111 (2019)

13. E. Scripnic, Stiinta Agricola 1, 66-69 (2013)

14. E.A. Miftakhutdinova, Theory and Practice of Meat Processing 2, 4-11 (2020)

15. D. Borda-Molina, Poultry Science 7, 2906-2918 (2019) 